\title{
Thermodynamical properties of metric fluctuations during inflation
}

\author{
Mauricio Bellini* \\ Instituto de Física y Matemáticas, \\ Universidad Michoacana de San Nicolás de Hidalgo, \\ AP: 2-82, (58041) Morelia, Michoacán, México
}

\begin{abstract}
I study a thermodynamical approach to scalar metric perturbations during the inflationary stage. In the power-law expanding universe here studied, I find a negative heat capacity as a manifestation of superexponential growing for the number of states in super Hubble scales. The power spectrum depends on the Gibbons-Hawking and Hagedorn temperatures.
\end{abstract}

During inflation vacuum fluctuations on scales smaller than the Hubble radius are magnified into classical perturbations of the scalar field on super Hubble scales. The primordial perturbations arise solely from the zero-point fluctuations of the quantized fields. Although the region which ultimately expanded to become the observed universe may have contained excitations above the vacuum, these excitations would not have any significant effect on the present state of the universe because a sufficiently large amount of the inflation would have redshifted these excitations to inmeasurably long wavelengths [1]. Hence, the density perturbations should be responsible for the large scale structure formation in the universe [3].

The scalar perturbations of the metric are related to density perturbations. These are the spin-zero projections of the graviton, which only exist in nonvacuum cosmologies. The issue of gauge invariance becomes critical when attempt to analyze how the scalar metric perturbations produced in the very early universe influence a globally flat isotropic and homogeneous universe on super Hubble scales. This allows us to formulate the problem of the evolution for the amplitude of scalar metric perturbations around the FriedmannRobertson-Walker (FRW) universe in a coordinate-independent manner at every moment in time 四]. Since the results do not depend on the gauge, the perturbed globally flat FRW metric is well described by

$$
d s^{2}=(1+2 \psi) d t^{2}-a^{2}(t)(1-2 \Phi) d x^{2},
$$

*E-mail address: mbellini@mdp.edu.ar 
where $a$ is the scale factor of the universe and $\psi, \Phi$ are the scalar perturbations of the metric. I consider the particular case where the tensor $T_{i j}$ is diagonal, which implies that $\Phi=\psi$ [5]. On the other hand I will consider a semiclassical expansion for the scalar field $\varphi(\vec{x}, t)=\phi_{c}(t)+\phi(\vec{x}, t)$, with expectation values $\langle 0|\varphi| 0\rangle=\phi_{c}(t)$ and $\langle 0|\phi| 0\rangle=0$. Here, $|0\rangle$ denotes the vacuum state [6]. Since $\langle 0|\Phi| 0\rangle=0$, the expectation value of the metric (1) gives the background metric that describes a flat FRW spacetime.

Linearizing the Einstein equations one obtains the system of differential equations for $\phi$ and $\Phi$ [3]

$$
\begin{aligned}
& \ddot{\Phi}+\left(\frac{\dot{a}}{a}-\frac{2 \ddot{\phi}_{c}}{\dot{\phi}_{c}}\right) \dot{\Phi}-\frac{1}{a^{2}} \nabla^{2} \Phi+2\left[\frac{\ddot{a}}{a}-\left(\frac{\dot{a}}{a}\right)^{2}-\frac{\dot{a}}{a} \ddot{\phi}_{c}\right. \\
& \frac{1}{a} \frac{d}{d t}(a \Phi)_{, \beta}=\frac{4 \pi}{M_{p}^{2}}\left(\dot{\phi}_{c} \phi\right)_{, \beta}, \\
& \ddot{\phi}+3 \frac{\dot{a}}{a} \dot{\phi}-\frac{1}{a^{2}} \nabla^{2} \phi+V^{\prime \prime}\left(\phi_{c}\right) \phi+2 V^{\prime}\left(\phi_{c}\right) \Phi-4 \dot{\phi}_{c} \dot{\Phi}=0 .
\end{aligned}
$$

The dynamics for the background field is given by the equations

$$
\begin{aligned}
& \ddot{\phi}_{c}+3 \frac{\dot{a}}{a} \dot{\phi}_{c}+V^{\prime}\left(\phi_{c}\right)=0, \\
& \dot{\phi}_{c}=-\frac{M_{p}^{2}}{4 \pi} H_{c}^{\prime}\left(\phi_{c}\right),
\end{aligned}
$$

where $H_{c}\left(\phi_{c}\right) \equiv \dot{a} / a$ and $M_{p}$ is the Planckian mass. Furthermore, the overdot denotes the time derivative and the prime represents the derivative with respect to $\varphi$, evaluated in $\phi_{c}$ [i.e., $\left.V^{\prime}\left(\phi_{c}\right) \equiv \frac{d V(\varphi)}{d \varphi}\right|_{\varphi=\phi_{c}}$ and $\left.V^{\prime \prime}\left(\phi_{c}\right) \equiv \frac{d^{2} V(\varphi)}{d \varphi^{2}}\right|_{\varphi=\phi_{c}}$ ].

The eq. (2) can be simplified by means of the map $h=e^{1 / 2 \int\left(\frac{\dot{a}}{a}-\frac{2 \ddot{p}_{c}}{\phi_{c}}\right) d t} \Phi$, and one obtains a Klein-Gordon equation for the redefined scalar metric fluctuations $h$ []]

$$
\begin{aligned}
& \ddot{h}-\frac{1}{a^{2}} \nabla^{2} h-\left\{\frac{1}{4}\left(\frac{\dot{a}}{a}-\frac{2 \ddot{\phi}_{c}}{\dot{\phi}_{c}}\right)^{2}+\frac{1}{2}\left(\frac{\ddot{a}-\dot{a}^{2}}{a^{2}}-\frac{2 \frac{d}{d t}\left(\ddot{\phi}_{c} \dot{\phi}_{c}\right)-4 \ddot{\phi}_{c}^{2}}{\dot{\phi}_{c}^{2}}\right)\right. \\
&\left.-2\left[\frac{\ddot{a}}{a}-\left(\frac{\dot{a}}{a}\right)^{2}-\frac{\dot{a}}{a} \frac{\ddot{\phi}_{c}}{\dot{\phi}_{c}}\right]\right\} h=0 .
\end{aligned}
$$

This field can be written as a Fourier expansion in terms of the modes $h_{k}=e^{i \vec{k} . \vec{x}} \xi_{k}(t)$

$$
h(\vec{x}, t)=\frac{1}{(2 \pi)^{3 / 2}} \int d^{3} k\left[a_{k} h_{k}+a_{k}^{\dagger} h_{k}^{*}\right],
$$

where $\xi_{k}(t)$ are the time dependent modes and $\left(a_{k}, a_{k}^{\dagger}\right)$ are the annihilation and creation operators which complies with the commutation relations $\left[a_{\vec{k}}, a_{\vec{k}^{\prime}}^{\dagger}\right]=\delta^{(3)}\left(\vec{k}-\vec{k}^{\prime}\right)$ and $\left[a_{\vec{k}}, a_{\vec{k}^{\prime}}\right]=\left[a_{\vec{k}}^{\dagger}, a_{\vec{k}^{\prime}}^{\dagger}\right]=0$. The commutation relation for $h$ and $\dot{h}$ is $[h, \dot{h}]=i \delta^{(3)}\left(\vec{x}-\vec{x}^{\prime}\right)$ which implies that $\xi_{k} \dot{\xi}_{k}^{*}-\dot{\xi}_{k} \xi_{k}^{*}=i$, for any time. Replacing eq. (8) in (7) one obtains the equation of motion for $\xi_{k}(t)$ 


$$
\begin{gathered}
\ddot{\xi}_{k}+\left\{\frac{k^{2}}{a^{2}}+2\left[\frac{\ddot{a}}{a}-\left(\frac{\dot{a}}{a}\right)^{2}-\frac{\dot{a}}{a} \frac{\ddot{\phi}_{c}}{\dot{\phi}_{c}}\right]-\frac{1}{4}\left(\frac{\dot{a}}{a}-2 \frac{\ddot{\phi}_{c}}{\dot{\phi}_{c}}\right)^{2}\right. \\
\left.-\frac{1}{2}\left(\frac{\ddot{a} a-\dot{a}^{2}}{a^{2}}-\frac{2 \frac{d}{d t}\left(\ddot{\phi}_{c} \dot{\phi}_{c}\right)-4 \ddot{\phi}_{c}^{2}}{\dot{\phi}_{c}^{2}}\right)\right\} \xi_{k}=0,
\end{gathered}
$$

which can be written in a simplified manner as $\ddot{\xi}_{k}+\omega_{k}^{2}(t) \xi_{k}=0$, where

$$
\omega_{k}^{2}=a^{-2}\left[k^{2}-k_{0}^{2}(t)\right]
$$

is the squared frequency with wavenumber $k$ and the effective parameter of mass is

$$
\mu^{2}(t)=\frac{k_{0}^{2}(t)}{a^{2}}=\frac{1}{4}\left(\frac{\dot{a}}{a}-\frac{2 \ddot{\phi}_{c}}{\dot{\phi}_{c}}\right)^{2}+\frac{1}{2}\left(\frac{\ddot{a} a-\dot{a}^{2}}{a^{2}}-\frac{2 \frac{d}{d t}\left(\ddot{\phi}_{c} \dot{\phi}_{c}\right)-4 \ddot{\phi}_{c}^{2}}{\dot{\phi}_{c}^{2}}\right)-2\left[\frac{\ddot{a}}{a}-\left(\frac{\dot{a}}{a}\right)^{2}-\frac{\dot{a}}{a} \frac{\ddot{\phi}_{c}}{\dot{\phi}_{c}}\right] .
$$

Here, $k_{0}(t)$ is the time dependent wavenumber which separates the infrared (IR) (with $k \ll k_{0}$ ) and ultraviolet (UV) (with $k \gg k_{0}$ ) sectors. The IR sector describes the super Hubble dynamics for the metric fluctuations which are responsible for gravitational effects on cosmological scales.

The issue of the increasing number of degrees of freedom for matter field fluctuations in the IR sector in the framework of thermodynamics was studied in another work [8]. The main aim of this work is the study of thermodynamical properties for scalar metric fluctuations on super Hubble scales. To make a thermodynamical description for these fluctuations we can write the partition function $Z(\beta)$ on super Hubble scales

$$
Z(\beta) \simeq \int_{k=0}^{\epsilon k_{0}} \frac{d^{3} k}{(2 \pi)^{3 / 2}} e^{-\beta \omega_{k}(t)}=\int_{k=0}^{\epsilon k_{0}} d \omega_{k} \rho\left(\omega_{k}\right) e^{-\beta \omega_{k}}
$$

where $\beta^{-1}$ is related to the background "temperature". The squared frequency with cut-off wavenumber $\epsilon k_{0}$ is given by $\omega_{\epsilon k_{0}}^{2}=-a^{-2}\left[k_{0}^{2}\left(1-\epsilon^{2}\right)\right]$, where $\epsilon$ is a dimensionless parameter given by $k / k_{0} \ll 1$. In the semiclassical limit the frequency $\omega_{k}$ plays the role of the energy for each mode with wavenumber $k$. In the IR sector the wavenumbers are very small with respect to $k_{0}\left(k \ll k_{0}\right)$, and the frequency $\omega_{k}$ is imaginary pure $\left(\omega_{k}= \pm i\left|\omega_{k}\right|\right)$. The function $\rho\left(\omega_{k}\right)$ gives the density of states with frequency $\omega_{k}$

$$
\rho\left(\omega_{k}\right)=\frac{1}{(2 \pi)^{3}}\left|\frac{d^{3} k}{d \omega_{k}}\right|=\frac{k^{2}}{2 \pi^{2}}\left|\frac{d k}{d \omega_{k}}\right|
$$

where $\left|\frac{d^{3} k}{d \omega_{k}}\right|$ is the Jacobian of the transformation such that $\left|\frac{d \omega_{k}}{d k}\right|=\frac{\left[k_{0}^{2}+a^{2} \omega_{k}^{2}\right]^{1 / 2}}{a^{2}\left|\omega_{k}\right|}$, where $\left|\omega_{k}\right|=$ $\left[\omega_{k} \omega_{k}^{*}\right]^{1 / 2}$ and the asterisk denotes the complex conjugate. Hence, the density of states with frequency $\omega_{k}$ can be written as

$$
\rho\left(\omega_{k}\right)=\frac{1}{2 \pi^{2}}\left[k_{0}^{2}+a^{2}(t) \omega_{k}^{2}\right]^{1 / 2}\left|\omega_{k}\right| a^{2}(t) .
$$


Energy added to a system can go either into increasing the energy of existing states or into creating new states. In the case of the IR gauge-invariant metric fluctuations new and new states are created from the UV sector during inflation. These fluctuations can be written as a Fourier expansion taking into account only the modes with $k \ll k_{0}$

$$
h_{c g}=\frac{1}{(2 \pi)^{3 / 2}} \int d^{3} k \theta\left(\epsilon k_{0}-k\right)\left[a_{k} h_{k}+a_{k}^{\dagger} h_{k}^{*}\right],
$$

where $\epsilon \ll 1$ is a dimensionless parameter and $k_{0}$ is the time dependent wavenumber that separates the IR and UV sectors. The inverse of "temperature" and the heat capacity are given by

$$
\begin{aligned}
\beta & =\left.\frac{\partial \ln [\rho]}{\partial \omega_{k}}\right|_{k=\epsilon k_{0}}, \\
C_{V} & =-\left.\left(\beta \beta^{*}\right)\left[\frac{\partial^{2} \ln [\rho]}{\partial \omega_{k}^{2}}\right]\right|_{k=\epsilon k_{0}} .
\end{aligned}
$$

The quantum nature of the metric fluctuations (in the UV sector) is the motivation for which $\beta$ is imaginary pure. If the second derivative in eq. (17) is positive, hence $C_{V}$ becomes negative and the density of states rises superexponentially. Systems with negative heat capacity are thermodynamically unstable. They are placed in contact with a heat bath and will experience runway heating or cooling. If the density of states grows exponentially, an inflow of energy at the Hagedorn temperature [9] goes entirely into producing new states, leaving the temperature constant. If the density of states grows superexponentially, the process is similar, but the production of new states is so copious that the inflow of energy actually drives the temperature down. In our case the Hagedorn temperature is not a true temperature. It takes into account the energy of each mode $h_{k}$.

To simplify de notation, I will denote $\omega_{\epsilon k_{0}}$ as $\omega$. During inflation the heat capacity for IR gauge-invariant scalar metric perturbations is given by

$$
C_{V}=\frac{-\mu^{4}\left(\omega^{2} \mu^{2}+\mu^{4}+2 \omega^{4}\right)}{\omega^{4}\left(\mu^{2}+\omega^{2}\right)^{4}},
$$

where $\mu^{2}=k_{0}^{2} / a^{2}>0$. The inverse of the effective "temperature" is

$$
\beta \simeq \mp i \frac{\mu^{2}}{|\omega|\left(\mu^{2}+\omega^{2}\right)} .
$$

Note that $\beta$ describes the environment of the IR sector. If $C_{V}>0$, the system distributes its energy in the existent states. The situation $C_{V}<0$ describes a system which increments very rapidly the number of states.

Now I consider the case of a power-law expanding universe for which the scale factor evolves as $a(t) \propto\left(t / t_{0}\right)^{p}$ and the Hubble parameter is $H_{c}\left[\phi_{c}(t)\right]=p / t$. The background field is given by $\phi_{c}(t)=\phi_{c}^{(0)}-m \ln \left[\left(t / t_{0}\right) p\right]$, where $\phi_{c}^{(0)}=\phi_{c}\left(t=t_{0}\right)$ and $t \geq t_{0}$. Furthermore, $m \simeq\left(10^{-4}-10^{-6}\right) M_{p}$ is the mass of the inflaton field. In this particular case $h(\vec{x}, t)=$ $t^{\left(\frac{p}{2}+1\right)} \Phi(\vec{x}, t)$ and $\mu^{2}=\frac{k_{0}^{2}}{a^{2}}$ being given by

$$
\mu^{2}(t)=M^{2} t^{-2}
$$


where $M^{2}=\left(p^{2}+4\right) / 4$. The density of states and $\beta$ are

$$
\begin{aligned}
\rho\left(\omega_{k}\right) & \simeq \frac{\left|\omega_{k}\right|}{2 \pi^{2}} t^{3 p}\left[M^{2} t^{-2}+\omega_{k}^{2}\right]^{1 / 2}, \\
\beta(t) & \simeq \mp \mathrm{i} \frac{t}{\epsilon^{2} M} .
\end{aligned}
$$

Finally, the heat capacity that results is

$$
C_{V} \simeq-2\left(\beta \beta^{*}\right)^{2} \simeq-2 \frac{t^{4}}{\epsilon^{8} M^{4}}
$$

which is negative but its absolute value increases as $t^{4}$. This is a manifestation of the superexponential growth of the number of degrees of freedom for the IR scalar metric fluctuations in a power-law expanding inflationary universe. The Hagedorn temperature $\left(\beta \beta^{*}\right)^{-1 / 2}$ go asymptotically to zero as $t^{-1}$. Both facts, $\dot{C}_{V}<0$ and $\left.\left[\beta \beta^{*}\right]^{-1 / 2}\right|_{t \rightarrow \infty} \rightarrow 0$, are consequence of the instability of the IR sector during inflation. Such an instability is due to the interaction of the inflaton field, which manifests itself in the temporal dependence of the effective parameter of mass $\mu(t) \sim t^{-1}$.

If $\left(\beta \beta^{*}\right)^{-1 / 2}$ is the zero mode "temperature" (or background temperature), the squared metric perturbations when the horizon entry will be given by

$$
\left\langle\Phi_{c g}^{2}\right\rangle_{I R}=\left.\int_{k=0}^{\epsilon k_{0}} \frac{d k}{k} \mathcal{P}_{\Phi_{c g}}(t) \simeq t^{-(p+2)} \int_{0}^{\epsilon k_{0}} \frac{d^{3} k}{(2 \pi)^{3}}\left[\xi_{k=0}(t)\right]^{2}\right|_{t_{*}},
$$

where $t_{*}$ is the time when the horizon entry and $\xi_{k=0}$ is the solution of $\ddot{\xi}_{0}-\left(\frac{\epsilon^{2}}{\beta \beta^{*}}\right) \xi_{0}=0$. The asymptotic solution for this equation is

$$
\xi_{0}(t) \simeq c_{1} t^{\frac{1}{2}\left[1+\sqrt{1+4 M^{2}}\right]},
$$

In other words (for $p>3.04$ needed to power-law inflation takes place), the power spectrum

$\left.\mathcal{P}_{\Phi_{c g}}\left(t_{*}\right) \simeq 2 \epsilon^{2}\left(t^{3 p+\sqrt{5+p^{2}}-1}\right)_{t_{*}} \frac{T_{H}^{2}}{T_{G H}^{2}(t)}\right|_{t_{*}} k_{*}^{3}$ is a function of the Gibbons-Hawking and Hagedorn temperatures, given respectively by $T_{G H}=H_{0} /(2 \pi)$ and $T_{H}=\left(\beta \beta^{*}\right)^{-1 / 2}$. Here, $T_{G H}$ is the temperature of the primordial horizon (with size $H_{0}^{-1}$ ) and $T_{H}\left(t_{*}\right)$ is the temperature related to the cosmic horizon at the moment of horizon-crossing [which has a size $H^{-1}\left(t_{*}\right)$ ].

To summarize, super Hubble metric fluctuations with negative heat capacity during power-law inflation describes superexponential growth of the number of states, which is a characteristic of nonequilibrium thermodynamical systems. Rather an increasing energy density, an increasing of $\left|C_{V}\right|$ (for $C_{V}<0$ ), gives a superproduction of the number of degrees of freedom in the infrared sector. Notice that the heat capacity in the model here studied decreases very rapidly. The imaginary nature of $\beta$ must be understood as a consequence of the quantum nature of the metric fluctuations in the ultraviolet sector, which plays the role of infrared's environment in the coarse-grained field $h_{c g}$-representation here developed.

\section{ACKNOWLEDGMENTS}

I would like to acknowledge CONACYT (México) and CIC of Universidad Michoacana for financial support in the form of a research grant. 


\section{REFERENCES}

[1] A. H. Guth, Phys. Rev. D23, 347 (1981).

[2] A. A. Starobinsky, in: Fundamental interactions, MGPI Press, Moscow, 1983;

A. A. Starobinsky, in: H. J. de Vega, N. Sánchez (Eds.), Current Topics in Field Theory Quantum Gravity, and Strings, Springer, New York, 1986.

[3] for review on inflation see: A. D. Linde, Particle Physics and Inflationary Cosmology, (Harwood, Chur, Switzeland, 1990); A. S. Goncharov, A. D. Linde, V. F. Mukhanov, Int J. Mod. Phys. A2, 561 (1987).

[4] A. S. Goncharov, A. D. Linde, V. F. Mukhanov, Int. J. Mod. Phys. A2, 561 (1987).

[5] V. F. Mukhanov, L. R. W. Abramo and R. H. Brandenberger, Phys. Rev. Lett. 78, 1624 (1997).

[6] M. Bellini, H. Casini, R. Montemayor, P. Sisterna, Phys. Rev. D54, 7172 (1996).

[7] Mauricio Bellin, Phys. Rev. D61, 107301 (2000).

[8] Mauricio Bellini, Phys. Rev. D63, 087301 (2001).

[9] R. Hagedorn, Nuovo Cimento, Suppl. 3, 147 (1965); ibid., Nuovo Cimento A56, 1027 (1968). 